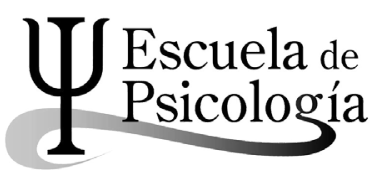

Wímb lu, Rev. electrónica de estudiantes Esc. de psicología, Univ. de Costa Rica. 10 (1): 67-81, 2015 / ISSN: 1659-2107

\title{
LA PSICOLOGÍA COMO CIENCIA ENSAYO DE REFLEXIÓN TEÓRICA CON ÉNFASIS EN COSTA RICA
}

\author{
Psychology as a science in Costa Rica. \\ Theoretical reflexing essay with an emphasis on Costa Rica
}

Cristina Paniagua Esquivel*

\begin{abstract}
Resumen: Más que una disciplina, la psicología se considera como un conjunto de ciencias, en donde el psicólogo se encarga de integrar los conocimientos (Henriques, 2011; Polo, 2009); también se considera como una disciplina dividida (Polo, 2009; Campos, 2013). Como disciplina que estudia al ser humano, se ha categorizado como ciencia social, entrando en la polémica que envuelve a estas ciencias, de si deben entenderse como una ciencia o no (Bernal, 2006). El objetivo de esta revisión es presentar un acercamiento básico, teórico, a la discusión que existe sobre si la psicología es o no ciencia, específicamente en Costa Rica. Se propone la investigación como eje fundamental en todas las áreas y la observación como el método unificador. Se destaca, en menor medida, la experimentación como el método que presenta la parte que se considera "más científica" de psicología, pero sin querer destacarlo como única vía. Se presenta la historia de la psicología (en general como en Costa Rica). También, las características de la ciencia. Finalmente, se considera que efectivamente es una ciencia, aunque las divisiones anteriormente mencionadas, debilitan su definición como tal.
\end{abstract}

Palabras clave: Psicología, Ciencia, Costa Rica, Ciencias Sociales, reflexión teórica.

Abstract: More than a discipline, psychology is considered as a group of sciences, in where the psychologist is in charge of the integration of knowledge (Henriques, 2011; Polo, 2009); is also considerate as a split discipline (Polo, 2009; Campos, 2013). As a discipline that studies humans, is categorized as a social science, bringing it inside the polemics around these sciences, if they must be seen as a science or not (Bernal, 2006). The objective of this review is to present a basic, theoretical approach, to the discussion around if psychology is or not a science, specifically, in Costa Rica. Research is presented as a transversal axis in all psychological areas and observation as an unification method. Also, experimental research is presents as the "most scientific" considerated part of psychology, but no to put it as the only way. Psychology history is presented (general and in Costa Rica). Also, science characteristics. Finally, psychology us considered as a science, bus, divisions early presenter, weakens its definition as one.

Key Words: Psychology, Science, Costa Rica, Social Science, theoretical reflexion.

Estudiante de psicología, Universidad de Costa Rica.

Correo electrónico: cpaniaguae@gmail.com

Recepción: 1/8/2014 Aceptación: 8/1/2015 


\section{Introducción}

La discusión de si la psicología debe ser considerada o no una ciencia, existe desde que se fundó el primer laboratorio a cargo primero de Wilhelm Wundt en 1879 y posteriormente William James en 1890 (Brock, 2007), y se mantiene hasta ahora. Está tanto en el mundo como en Costa Rica, a pesar de que desde sus inicios se ha adherido a los métodos experimentales y científicos, y en la actualidad, siguen presente en su formación. Entonces, ¿Qué hace que se dude de su cientificidad?

Johann Herbart (1776-1841) afirmó que la psicología es una ciencia, y ciencia autónoma, no subordinada a la filosofía ni a la fisiología (Polo, 2009). Esta cita es relevante, considerando que su autor, murió antes de que se fundara oficialmente el primer laboratorio de investigación en psicología

En contraposición a esta afirmación, está Polo (2009), quien cuestiona la posición de la psicología como ciencia, ya que afirma que no aparenta tener un objeto de estudio - definido como la materia de conocimiento que estudia una ciencia (Gallo, 2000) -, sino que toma de otras disciplinas lo requerido y se preocupa por tratar de armar un ser humano integral a partir de ese conocimiento adquirido por otros. Sin embargo, la psicología tiene objeto de estudio: comprender al sujeto humano (Hernández-Gómez, 2011).

En una sociedad en donde la etiqueta "ciencia" hace que lo que se le asocie sea altamente valorado y se le brinde una alta fiabilidad (Chalmers, 2003), la psicología busca entrar en esa categoría, precisamente para tener ese valor y esa estima. Esto se torna relevante, cuando se ven movimientos que buscan, por ejemplo, negar la existencia de conceptos como enfermedad mental (Szasz, 2001) o incluso de mente; conceptos asociados con psicología. No se profundizará en estas discusiones, pero sirven de ejemplo del cuestionamiento de la validez del objeto de estudio de la psicología.

El presente ensayo tiene como objetivo revisar la discusión que existe sobre si la psicología es o no ciencia, bajo la premisa de que lo es y que en Costa Rica, se ha desarrollado como tal desde el inicio. Existen autores que han discutido sobre esta temática, y este artículo pretende ser una reflexión en el contexto costarricense.

El documento toma como inicio, la definición de ciencia y sus características, para entender la psicología como tal. También es necesario entender qué es psicología, de dónde viene, cómo nació y cómo es la psicología en Costa Rica desde sus inicios. Finalmente, se retomarán dos métodos en la ciencia: Observación y experimentación, y se desarrollará su relevancia en la psicología. A modo de cierre se harán reflexiones finales, para buscar una respuesta a si la psicología en Costa Rica es o no una ciencia. 


\section{Ciencia}

\section{¿Qué es la ciencia?}

Históricamente ha existido una polémica sobre si las ciencias sociales dentro de la cual está la psicología- son ciencia (Bernal, 2006).

Cuando se habla de ciencia y de hechos científicos, las personas asumen la existencia de fiabilidad, de verdad, y se otorga un alto valor a aquello que lleve estas etiquetas. Chalmers (2003) afirma que el sólo hecho de que algo sea denominado científico, hace que se le otorgue un lugar importante y una alta valoración.

Según Bunge (1960), la ciencia es un grupo de ideas creadas por el ser humano que le permiten entender e interpretar el mundo en el que vive. Para crear un concepto de mundo, las personas que hacen ciencia recurren a la investigación científica, la cual genera un conocimiento racional, sistemático, exacto, verificable y fiable.

Otra característica de la ciencia es que el conocimiento que genera, el conocimiento científico, parte de los hechos (Bunge, 1960). El método científico, inicia con la teoría y recolecta los datos para apoyar o refutar esta teoría (Creswell, 2002). Este proceso de recolección genera afirmaciones del mundo, que se verifican con el uso de los sentidos (ver, oír, tocar) de manera objetiva, y que no intervienen las opiniones personales (Chalmers, 2003) ni valores emocionales o comerciales (Bunge (1960).

\section{Objetividad y subjetividad en la ciencia}

En ciencia, se requiere que los hechos sean obtenidos de manera objetiva. Este requerimiento proviene de corrientes positivistas y postpositivistas, que buscan que el conocimiento se desarrolle con base en observaciones y medidas cuidadosamente realizadas, con medidas numéricas, para que, según esta perspectiva, exista seguridad de que lo que se reporte "existe realmente en el mundo" (Audi, 2004; Creswell, 2002) y no que se deriven de lo que el científico piensa (Audi, 2004).

Del otro lado, se encuentra el subjetivismo, que refiere a aquellos enunciados que representan los sentimientos, no hechos. Se considera contrario al objetivo, ya que son válidas opiniones alternativas (Audi, 2004) que incluyan pensamientos o sentimientos de la persona que presenta el resultado o hecho y no se basa en una realidad existente.

Existe un concepto primordial en la objetividad, denominado intersubjetividad. Cuando un hecho/objeto se da o existe de una manera intersubjetiva, es porque todas las personas -o todas las personas de un mismo grupo- reconocen su 
existencia, a pesar de que no necesariamente exista independientemente de eso (Audi, 2004).

Esto es relevante, ya que algunos actores defienden que lo más cercano a la objetividad, es la intersubjetividad, en especial en investigaciones en Ciencias Sociales, en donde es imposible captar adecuadamente todos los comportamientos subjetivos de manera objetiva (Adorno, 2001).

\section{Psicología}

\section{Antecedentes de la psicología}

Los inicios de la psicología se remontan al Siglo XIX, cuando se separó de la filosofía; específicamente de la filosofía de la mente, la epistemología y la ética. De la ética, se desprenden áreas que buscan trabajar las motivaciones, la persona como ser social y temas similares. De la filosofía de la mente, que trataba las cuestiones de la existencia del alma, heredó la búsqueda de la existencia de la mente. De la epistemología, se desprende la búsqueda de cómo aprenden las personas, cómo se adquiere el conocimiento, percepción, memoria y pensamiento (Hardy, 1998).

Existen también tres movimientos científicos que se consideran fundamentales en la fundación de la psicología (Brennan, 1999): el primero son los progresos en la fisiología, -específicamente, en la fisiología del sistema nervioso- que en esa época establecen este sistema como el enlace entre el mundo y la mente. El segundo, es la psicofísica, que es la vertiente de la fisiología de los sentidos que destacaba la experiencia subjetiva, dentro de las relaciones entre los estímulos y las sensaciones.

Finalmente, se presenta la evolución. En este caso, se ven los trabajos de H. Spencer y F. Galtosm; el primero desarrolló una teoría sobre la asociación de sentimientos e ideas y su relación con la herencia y el segundo, desarrolló la idea de la existencia de ciertos rasgos, que son heredados y además medibles pos medio de pruebas y metodologías específicas (Brennan, 1999).

\section{¿Qué es la psicología?}

La palabra psicología viene del latín "psycbe logos", traducido como estudio de la psique; del alma (Hardy, 1988). Sin embargo, la actual psicología ha ido más allá del estudio del alma o la psique, y se ha convertido en una disciplina dividida. La Real academia española (2014), por ejemplo, muestra distintas definiciones, dentro de las que destacan que psicología que es parte de la filosofía, que se relaciona con el espíritu, que estudia los procesos mentales; la forma de sentir de 
una persona o de un pueblo -así como sus caracteres espirituales y morales - y finalmente, habla de la conducta animal.

Definir qué es psicologíicadocomplejo. Esta dificultad de definición responde a que bajo el concepto, existen muchas áreas. Cuando se pide a un estudiante o profesional en psicología que la defina, resalta un área y rescata que existen otras (Henriques, 2011). Un ejemplo de la división en psicología, se encuentran autores como Polo (2009), quien divide la psicología en dos: el psicoanálisis y la psicología cómo ciencia. Y Campos (2013), que explica que hay una lucha entre dos grandes "paradigmas", que son la terapia conductual y el psicoanálisis. A estos se le suman otros, como el humanismo (también llamado tercera fuerza), la psicología cognitiva y recientemente, enfoques como la neuropsicología.

Sin embargo, todos tienen un enfoque común: el ser humano. Una posible definición de psicología podría ser: La disciplina que estudia al ser humano. Sin embargo, esta definición es muy amplia y podría abarcar otras disciplinas, como las ciencias médicas u otras ciencias sociales.

Otra alternativa podría ser: La disciplina que estudia el desarrollo del ser humano de manera integral: mental, social, conductual, etc.; sea individualmente, en pareja, grupo o comunal, en un contexto social específico. El estudio del ser humano puede verse complementado con modelos animales. En esta definición, se rescata la existencia de estudio de las personas aún en contextos clínicos (en otras palabras, se considera que aún en psicoterapia, se hace un estudio de la persona o personas con las que se está trabajando).

Con una definición que trata al ser humano como un ente integral, permite que cada área de la psicología estudie una parte diferente de la persona. Así, quienes trabajan, por ejemplo, en investigación de procesos cognitivos, aportan al desarrollo mental y quienes trabajan en psicoterapia grupal, aportan al desarrollo social.

\section{Breve historia de la psicología}

Desde los inicios de la psicología, los primeros psicólogos consideraban que existía una relación entre los procesos psicológicos, y los fisiológicos (Wright, 1994, en Hardy, 1998). Entre 1876 y 1919, Wilhelm Wundt, considerado el fundador de la psicología, trabajó en su laboratorio la mente de una manera objetiva, mediante experimentos controlados (Brock, 2007; Hgothersall, 1997).

Este autor comenzó con un curso sobre psicología fisiológica y creo el primer laboratorio dedicado por completo a la investigación psicológica (Brennan, 1999). Al inicio, se enfrentó a críticas de colegas (presentes actualmente) que cuestionaban la legitimidad de la psicología como ciencia experimental. Sin embargo, el laboratorio creció y la literatura que desarrolló buscaba ser un punto intermedio entre la fisiología y la filosofía (Hothersall, 1997). 
Posteriormente, en 1890 inicia un proceso de expansión de la psicología en Estados Unidos, por los trabajos de William James (Brock, 2007). Proponía que lo físico y lo mental son parte de una experiencia, en distintas facetas y propone a la psicología como una ciencia de la vida normal (Brennan, 1999).

La psicología siguió evolucionando, y tuvo un nuevo viraje a mediados de 1959, considerada la fecha de nacimiento de la ciencia cognitiva (y la psicología cognitiva). En ese periodo, se realizó el Simposio sobre la Teoría de la Información, en el Instituto de Tecnología de Massachusetts (Segovia, 2012). En él se reunieron muchos grandes hombres de esa época en materias de comunicación y ciencias humanas, como Noam Chomsky, Herbert Simon y Marvin Minsky. Previo a esto, en 1948, en el Simposio de Hixón, un grupo de científicos se reunieron y presentaron temas que abarcaban la mente, el cerebro y los procedimientos lógicos.

Posterior a 1970, las investigaciones sobre la mente comienzan a sistematizarse; se desarrollan investigaciones para programar un ordenador para que procese la información igual que una mente, para entender cómo funciona el sistema cognitivo (Audi, 2004).

En la actualidad, se llama ciencia cognitiva a un conjunto de disciplinas que estudian la actividad inteligente, tanto en seres vivos como en máquinas. Se divide en dos áreas, la psicología cognitiva y la inteligencia artificial, y recibe contribuciones de otras disciplinas como la neurociencia, ciertas ramas de psicología y la filosofía (Audi, 204).

Este viraje es relevante, ya que los primeros psicólogos buscaban métodos para confirmar su hipótesis de que los procesos psicológicos pudieran relacionarse con los fisiológicos. Sin embargo, durante la mayor parte del Siglo XX, la disciplina se desvió (Wright, 1994, en Hardy, 1998). En la actualidad, y posterior a este nacimiento de la psicología cognitiva, se ha abierto en la disciplina la posibilidad de ligarse de nuevo con la fisiología. Este proceso ha llevado al estudio del sistema nervioso central, y al nacimiento de la neuropsicología, por medio del trabajo -mayormente- en sujetos humaionesexisten también modelos animales) para entender cómo opera el sistema cognitivo (Audi, 2004).

\section{La psicología en Costa Rica}

En Costa Rica, la psicología tuvo en sus primeros años dos vertientes: la salud mental y la investigación.

En 1957, en el Hospital Chapuí (Actualmente Hospital Nacional Psiquiátrico) dio inicio el servicio de psicología clínica, con el Dr. Gonzalo Adís a la cabeza de este (Adís, 1991; Campos, Pérez \& Rosalba, 1990). Se dio en una época en la que la salud mental tenía un viraje importante. A partir de ahí, comenzaron a ingresar al país diversos psicólogos, los cuales se entrenaron en este servicio de psicología como psicoterapeutas (Adís, 1991). A pesar de que existían cursos de psicología 
en diversas disciplinas, no fue hasta 1966 que se creó en la Universidad de Costa Rica la Escuela de psicología (Adís, 1991; Flores, 2010).

En lo que respecta a la investigación, en 1963 se funda el Centro de Investigación en Psicología (Actualmente el Instituto de Investigaciones Psicológicas) (Adís, 1991; Campos et al., 1990). Tres años después, en 1966, se inició en Costa Rica, en la Sección de Ciencias del Hombre de la Facultad de Ciencias y Letras de la Universidad de Costa Rica, se crea el bachillerato en psicología. En 1976, el entonces Departamento de Ciencias del Hombre se dividió en dos, y se creó oficialmente, la Escuela de Psicología, en la recién formada Facultad de Ciencias Sociales (Escuela de psicología de la Universidad de Costa Rica, 2013).

El énfasis del programa de estudio fue, desde sus inicios, formar a los psicólogos en técnica y en aplicaciones prácticas. A partir de los años 80, las investigaciones comenzaron a aumentar y en esa década, se crearon tres revistas y una maestría con énfasis en investigación (Campos et al., 1990) y a inicios de los años 90, se insistía en poner más énfasis en la investigación (Adís, 1991).

Esta descripción del programa de estudios de la carrera de psicología es relevante, ya que, como la primera escuela de psicología del país, marca la pauta de que los profesionales, tienen una formación científica.

La psicología en Costa Rica se ha marcado por procesos políticos, sociales y económicos, que provocaron que tuviera un crecimiento rápido, pero desordenado, en donde muchas veces se genera una contradicción entre las necesidades del Estado, y los objetivos de la disciplina (Campos et al., 1990). Estos movimientos se unieron a un proceso a nivel latinoamericano, en el que se cuestionan las Ciencias Sociales, y en el caso de psicología, sus enfoques teóricos y técnicos. Producto de esto, se desarrolló una psicología más crítica en su rol a nivel nacional (Flores, 200).

\section{¿Es psicología una ciencia?}

Para poder entender a la psicología como ciencia, se desarrolló una definición de ciencia, con sus características y se dio una breve historia de psicología, tanto a nivel mundial como en Costa Rica. A través del desarrollo de los apartados, se fue adelantando algunas afirmaciones que se apegan a la premisa de que psicología es una ciencia. Sin embargo, a continuación se desarrollarán dos de los métodos que más se han visto representados en la ciencia, y la manera en que se desarrollan en psicología: experimentación y observación. Finalmente, se hará referencia a un tema que es también relacionado con el desarrollo de lo que es psicología como ciencia, que es la existencia de paradigmas (desde la teoría de Thomas Kuhn) en psicología. 
Se ha escrito mucho sobre esta temática (especialmente sobre observación), por lo cual este apartado busca ser una síntesis de discusiones sobre estos temas.

Se han desarrollado dos características de la ciencia, que son la experimentación y la observación, que corresponden a dos ejes importantes que están presentes en la psicología. También, se trabajó el concepto de paradigma de Kuhn, el cual se ha ligado con las Ciencias Sociales. A continuación, se da una visión de estos tres conceptos desde la visión de la psicología.

\section{La observación en psicología}

En psicología, la observación es fundamental. Existe literatura especializada en el método observacional, para que sea estructurada, y que los diseños permitan la categorización, la adecuada recolección de datos y el mejor muestreo (Ver, por ejemplo, los trabajos de María Teresa Anguera y Vincen ç Quera). Y no es importante únicamente para la psicología; es considerada como inseparable de las actividades en la ciencia, ya que acompañan todo el proceso de creación de conocimiento (Riba, 1993). Es además, el método más antiguo de recolección de datos. Cuando hablamos de observación como método, se hace referencia a la observación científica, la cual es más analítica, objetiva y requiere de hipótesis.

Dentro de la literatura, se defiende que la observación tiene ciertas ventajas. Por ejemplo, se obtiene la información como ocurre, se da información que los sujetos de estudio no pueden proporcionar y requiere menos cooperación activa de los sujetos (Anguera, 1993). Como método, la observación va más allá de la recepción de estímulos visuales, y que requiere estar orientado por la teoría y nuestros conocimientos previos (Riba, 1993; Chalmers, 2003) controlando que estos conocimientos no sean fuente de sesgo; por ende, que sean objetivas.

Para que una observación sea considerada objetiva y válida, debe tener la posibilidad de ser probada por procedimientos directos y falibles, que permitan ser evaluados conforme los adelantos en la ciencia y la tecnología se vayan presentando (Chalmers, 2003). Es ahí donde entra el uso de elementos para el análisis, como protocolos estructurados, videocámaras y una base teórica que permita un sistema estructurado y claro de categorías. Además, de la existencia de software especializados para garantizar la calidad de la observación.

Sin embargo, también existen limitaciones, como el hecho de que es imposible predecir todos los sucesos y determinar la duración de los sucesos. Un elemento importante que se destaca es que muchos autores manejan la idea de que no es cuantificable la información obtenida por medio de la observación, sin embargo, Anguera (1993) establece que es posible.

La especialización de la observación en psicología, ha permitido que existan técnicas para validar las categorías observadas, por medio de distintas teorías, y la fiabilidad. 


\section{Experimentación}

Por otro lado, de la mano de la observación, está la experimentación. Se destaca la experimentación en psicología como el método que se usa en la ciencia, asociado al jipotético-deductivo (en el que se toma una hipótesis y se comprueba, para deducir de ella, conclusiones; Carvajal \& Matamoros, 2012). No se quiere decir, con esto, que sea la única forma de investigación (en general ni en psicología) ni que otros métodos (tanto en lo que se considera investigación cuantitativa como en modelos mixtos y cualitativos) tengan menor valor.

Según Gallo (2000) el experimento es un método para descubrir la relación entre dos o más principios, de manera controlada y que se da en condiciones objetivas previamente establecidas. La ciencia utiliza los experimentos como parte del método científico, en donde se debe hacer un test a las hipótesis generadas para poder verificarlas (Bunge, 1960). Las conclusiones se dan a partir del proceso de experimentación. Los experimentos dependen de un marco teórico, ya que la aceptación de los resultados de estos dependerá de la teoría. Además, si el conocimiento detrás de un experimento es deficiente o erróneo, los resultados también los serán (Chalmers, 2003).

Desde los inicios de la psicología, la experimentación ha sido un elemento clave. Los primeros psicólogos nacieron en un laboratorio experimental, ligados a la fisiología y el estudio empírico de la mente. Esta tradición de experimentación, se mantuve con la corriente conductista liderada principalmente por John Watson, Ivan Pavlov y Burrhus Skinner (Polo, 2009).

Finalmente, según el autor Thomas Kuhn (2004), lo que es ciencia o no ciencia está determinado por una comunidad científica. Este autor define que la ciencia normal es investigación, que se basa en una o más realizaciones científicas, reconocidas por una comunidad científica como fundamento para su práctica.

El principal objetivo de la psicología experimental, es acercar a la psicología a las ciencias duras (Mandler, 2007). Este proceso se desarrolló fuertemente, durante el Siglo XX, con los experimentos relacionados con los modelos animales y el condicionamiento, producto de la psicología conductista (Polo, 2009).

El proceso de cambiar la psicología de una filosofía racionalista a una ciencia empírica, tuvo un éxito reflejado en la creación de laboratorios para la psicología experimental en universidades de todo el mundo, pero principalmente en los Estados Unidos y en Alemania (Polo, 2009). Del experimento de la conducta, se procedió al experimento con la cognición y los procesos sociales.

Para que la psicología fuera considerada experimental con Wundt en 1974, requirió que se propusiera que sólo existe un tipo de explicaciones causales en psicología, que manipularan las condiciones externas en el experimento, y que los productos mentales como los pensamientos complejos fueran relacionados con observación subjetiva (Polo, 2009). 
La experimentación en psicología ha evolucionado, y ha enfocado su objeto de procesos conductuales, a procesos mentales, como la memoria, o los procesos mentales. Las teorías que desarrollan conceptos relacionados con el funcionamiento de procesos mentales y el acercamiento fisiológico al sistema nervioso central, han permitido que se desarrollen experimentos que evalúan esos procesos de manera sistemática.

\section{Paradigmas en psicología}

Finalmente, es relevante discutir brevemente un argumento que se ha utilizado numerosamente, para justificar a la psicología como ciencia, y es la existencia de paradigmas dentro de esta. Esto se argumenta por la relación entre las ideas Thomas Khun (quién desarrolló la teoría de paradigmas en la ciencia) y Ciencias sociales, al presentar una posición que se aleja del positivismo -principalmente en la búsqueda de fundamentos universales (Campos, 2013), lo cual no es del todo cierto en psicología.

Los paradigmas se consideran como realizaciones científicas, que son universalmente reconocidas durante un tiempo y que, durante ese tiempo, dan modelos de problemas y soluciones a la comunidad científica (Kuhn, 2004).

Los paradigmas en la ciencia tienen dos características: atraen a un grupo duradero de partidarios, a la vez que son lo bastante incompletos como para que los científicos los resuelvan y completen (Kuhn, 2004). Los paradigmas tienen leyes, supuestos teóricos, instrumentos, técnicas y formas de aplicación de las leyes (Chalmers, 2003) así como las formas de resolver problemas (Kuhn, 2004).

Una vez aceptado el paradigma, los científicos continúan con las nuevas reglas que implica la nueva teoría.

Sin embargo, se han hecho críticas sobre el uso de este término en psicología. Campos (2013), como uno de los más recientes, establece que psicología no tiene características de paradigma, y que, sin embargo, se maneja el discurso de que psicología es una ciencia multiparadigmática.

El concepto de multiparadigma se presenta ya que dentro de psicología, existen distintas posiciones y escuelas, principalmente en los programas de trabajo clínico. Además, plantea que los textos de Kuhn dejan ver que existe la posibilidad de que él dudara de la cientificidad de la psicología, contradiciendo de esta manera los argumentos que defienden esta idea.

En psicología, en lugar de paradigmas, se propone hablar de pluralidad de corrientes de pensamiento, que son distintas en lo ideológico y epistemológico, pero no mutuamente excluyentes. De esta forma, Campos (2013) pone en duda si, en psicología, se puede hablar de ciencia desde Kuhn y su concepción de ciencia normal. 
En lo que refiere a esta pluralidad de corrientes o ramas de la psicología, Carvajal y Matamoros (2012) realizan un análisis de publicaciones en la Revista Costarricense de Psicología (revista oficial del Colegio Costarricense de Psicólogos, que presenta profesionales de todo el país). Y se destacan nueve áreas: la psicología social, la psicología educativa, la psicología clínica, la psicología organizacional, la neuropsicología clínica, la psicología de la salud, la psicobiología y la psicología forense.

La relevancia de esta citación es que, no solo da una posible división en áreas, sino que revela cómo, en sus diversas ramas, la psicología busca el desarrollo de la disciplina a través de la investigación y distintos métodos. Estos mismos autores, encuentran que la investigación científica permite el desarrollo profesional y académico de la psicología como disciplina.

\section{Discusión}

La psicología podría definirse como una disciplina que tiene como objeto de estudio al ser humano de manera integral, y que, a pesar de que existan distintas formas de abordar al ser humano (como ser social, su psique, sus cogniciones, su comportamientos, etc.) en distintos ámbitos (educación, clínica, investigación, laboral, entre otros) su objetivo es siempre entender qué hay detrás de lo que sucede con las personas. En ocasiones, es para entender más a profundidad un fenómeno, o una acción. En otras, es para poder hacer modificaciones, para ayudar a la persona o las personas a alcanzar un objetivo, o para mejorar un sistema (de trabajo, o escolar, o comunitario, por ejemplo).

Estas distintas formas de abordar al objeto, han creado una ruptura en la psicología, que ha llevado a denominar las posturas como paradigmas, dándole así un carácter de irreconciliable a estas diferencias. Algunas de estas áreas, luchan por el reconocimiento de la psicología como una ciencia.

El entrenamiento del psicólogo en la academia tiene diversos matices, y tiene como eje transversal a través de toda su formación, la investigación, con el objetivo de formar profesionales críticos y científicos. Entre sus posturas destacan: trabajar de manera objetiva, con un objetivo claro, haciendo una ciencia que tenga utilidad. Además, aboga por tener resultados verificables, siguiendo un método claro y congruente con el problema de investigación planteado.

Este eje transversal en psicología, se propone como un punto de encuentro en las distintas áreas. No en todas las áreas, se establecen estos puntos de manera tan concisa; esto no implica que no existan. La investigación científica en psicología ha permitido dar credibilidad a muchas áreas. Esto no debe tomarse como una descalificación a aquellas que declaran ser ajenas a los métodos más "rigurosos", sino un llamado a observar que siguen cumpliendo el objetivo fundamental de la psicología: el estudio del ser humano. 
La observación y la experimentación que son fundamentales en la ciencia, lo son también en psicología. La observación ha sido desarrollada hasta convertirse en un método fiable y verificable al relacionarse con la teoría adecuada. Se presenta en todos los ámbitos de psicología y se ha convertido en su principal medio de adquisición de información. No siempre con protocolos validados os en investigaciones, pero sí en experiencia diaria de muchos profesionales.

La experimentación dio inicio a la psicología como disciplina independiente de la filosofía y, posteriormente, de la fisiología; aunque posteriormente, se volvió a unir con esta última con el nacimiento de la neuropsicología. La experimentación puede darse en cualquier área de psicología, aunque en muchas ocasiones, el problema de investigación no puede ser resuelto por medio de los criterios experimentales. Es aquí en donde otros métodos (de corte mixto o cualitativo) obtienen un alto grado de validez.

Durante la definición de ciencia, se establecieron las características de esta. Hay áreas de la psicología que buscan cumplir muchas de estas, y las cumplen. ¿Qué hace que se dude de su cientificidad? Principalmente la respuesta está en la división.

Al ser el objeto de estudio de la psicología un sujeto igual que el psicólogo, se cuestiona si le es posible al profesional desprenderse de lo personal para poder observar la realidad de quien tiene en frente de manera real. En otras palabras, se cuestiona si existe una verdadera objetividad en su trabajo, debido a que los pensamientos y sentimientos del psicólogo pueden verse reflejado en lo que observa, o, en sus resultados experimentales. Esto impediría que se logre aprehender realmente la realidad.

Es en esta crítica que la intersubjetividad toma tanta relevancia, ya que permite que lo real, que no puede ser tomado por completo, sea mostrado por medio de la rigurosidad, y validado por una comunidad. En psicología, se plantea que el ser humano es un todo, pero en realidad no puede ser estudiado como tal. El estudio de áreas del ser humano, permite el estudio más riguroso de cada "parte". Partiendo de la inexistencia de la objetividad absoluta, la intersubjetividad es acertada para el desarrollo de conocimiento científico, que no se basa en lo que el investigador o profesional asume como adecuado.

Es importante destacar que, este estudio se puede hacer desde distintas áreas. No es necesario un laboratorio o un lugar de investigación para poder estudiar a la persona. La clínica (independientemente del modelo teórico y/o técnico), por ejemplo, permite el entendimiento del ser humano desde distintos ángulos, sin ser este necesariamente mutuamente excluyentes o contradictorios.

Críticos de la subjetividad en psicología asumen que, al ser un objeto de estudio tan cambiante, no se pueden hacer leyes como exigen algunas posturas de la ciencia, sino generalidades. Estas generalidades existen y pueden verse reflejadas en, por ejemplo, constructos clínicos. ¿Cómo diferenciamos una 
persona que padece de depresión de una que padece un ataque de ansiedad si no es porque se ha establecido, por medio de investigación, los criterios diagnósticos?

Entonces el objeto de estudio varía, pero individuos con ciertas características son agrupables, analizables. Se pueden generalizar resultados hechos de una manera óptima. Impresiona que, la conclusión principal sea que, dentro de la psicología hay ciencia, aunque la psicología como un todo no es ciencia. No implica que las áreas que no cumplen los criterios científicos, por ejemplo, las terapias psicodinámicas, deban ser eliminadas. Son formas de abordar al ser humano, que se complementan con aquellas que se ajustan a las características de la ciencia.

Vale preguntarse si, un alto porcentaje de lo que es psicología se apega a una definición actual de ciencia, sería posible establecerla como tal. Considerando la definición en donde en la psicología hay investigación siempr, y el enlace de la investigación con la ciencia, entonces la respuesta sería sí. Psicología es una ciencia, siempre que se mantenga de manera integral, no en las divisiones que se encuentran los mismos psicólogos en crear para desacreditarse unos a otros como psicólogos. Esto es una característica que tiene la psicología como disciplina joven, con pasado antiguo.

Para terminar, una última reflexión: antes de poder afianzarse como una ciencia de manera definitiva, la psicología deberá borras las líneas que desconectan a las áreas, ya que estas, en lugar de fortalecerlas, provoca una "Visión deformada de la psicología" (Campos, 2013, p. 24) que le impide crecer y posicionarse.

\section{Referencias bibliográficas}

Adís Castro, G. (1991). Sobre la historia de la psicología clínica en Costa Rica. Revista Cúpula, (23). 30-34.

Adorno, T. (2001) Epistemología y ciencias sociales (Vicente Gómez, trad.). Madrid: Frónesis. (Obra original publicada en 1975).

Audi, R. (Ed.) (2004). Diccionario Akal de filosofía (Humberto Marraud y Enrique Alonso, trads.). Madrid: Ediciones Akal. (Obra original publicada en 1999).

Bernal Torres, C.A. (2006). Metodología de la investigación (2da ed.). México: Pearson Prentice Hall.

Brennan, J.E. (1999). Historia y sistemas de la psicología (5ta. Ed.) (José Francisco Dávila Martínez, trad.). México: Prentice Hall. (Obra original publicada en 1997). 
Brock, A. C. (2007). Internationalizing the History of Psychology. New York: New York University Press (NYU Press).

Bunge, M. (1960). La ciencia, su método y su filosofía. Buenos Aires: Editorial Siglo Veinte.

Campos Santelices, A. (2013). ¿Paradigmas o paradigmatitis? acerca de los inconvenientes usos de este concepto en la epistemología psicológica. Rev. Ciencias Sociales, (139). 13-26.

Campos Ramírez, D.; Pérez Sánchez, R. \& Rosabal Coto, M. (1990). Identidad profesional e institucionalización de la psicología en Costa Rica: Crítica e interpretación histórica. Ciencias Sociales, (47). 81-91.

Carvajal, E. \& Matamoros, L. (2012). Análisis bibliométrico de la Revista Costarricense de Psicología, periodo 2001-2011. Revista Costarricense de Psicología, 31(12). 1-20

Chalmers, A. (2003). ¿Qué es esa cosa llamada ciencia? (3era ed.; Siglo veintiuno de España, trad.). México: Editorial Siglo XXI. (Obra original publicada en 1999).

Creswell, J. (2002). Research Design. Qualitative, quantitative, and mixed methods approaches (2da edición). California: Sage Publications.

Escuela de psicología de la Universidad de Costa Rica (2013). Historia de la escuela de psicología. En: http://www.psico.ucr.ac.cr/ig_historia.html

Flores Mora, D. (2010). La psicología en la Universidad de Costa Rica. 35 aniversario de la facultad de Ciencias Sociales. Rev. Reflexiones, 89 (1). 223-226

Gallo, R. (2000). Diccionario de la ciencia y la tecnología. México: Universidad de Guadalajara.

Hardy, T. (1998). Historia de la psicología. Principales corrientes en el pensamiento psicológico. Madrid: Prentice Hall

Heriques, G. (2011). A new unified theory of psychology. New York: Springer.

Hernández-Gómez, L. (2011). Una mirada crítica a la psicología moderna. Análisis sobre sus conceptos fundamentales. Revista De Psicología GEPU, 2(1), 104-116.

Kuhn, T. S. (2004). La estructura de las revoluciones científicas (Agustín Contín, trad.). México: Fondo de Cultura Económica [Obra original de 1962]. 
Mandler, G. (2009). History of Modern Experimental Psychology: From James and Wundt to Cognitive Science. Massachusetts: MIT Press

Polo Barrena, L. (2009). Curso de psicología general: lo psíquico: la psicología como ciencia: la índole de las operaciones del viviente (2a. ed.). España: EUNSA.

Real academia española. (2014). Psicología. En: http://lema.rae.es/ drae/?val=psicologia.

Riba, C. (1993). El método observacional. Decisiones básicas y objetivos. En M.T. Anguera (Ed), Metodología observacional en la investigación psicológica (Vol. I). Barcelona: Promociones y Publicaciones Universitarias, PPU

Segovia Cuellar, A. (2012). La cognición como acontecer biológico desde la teoría de la enacción y la corporización de la actividad psicológica. (Tesis para optar por el grado de Licenciatura). Universidad Nacional de Colombia, Colombia. Recuperado de http://www.bdigital.unal.edu.co/9152/1/ andressegoviacuellar.2012.pdf

Szasz, T. (2001). Ideología y enfermedad mental (Leandro Wolfson). Buenos Aires: Amorrortu Editores.

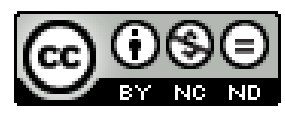

Este obra está bajo una licencia de Creative Commons ReconocimientoNoComercial-SinObraDerivada 4.0 Internacional. 